\title{
Gestión del conocimiento y su influencia sobre el desempeño organizacional en las empresas de Aguascalientes
}

\author{
Knowledge management and its influence on organizational \\ performance in Aguascalientes companies
}

\begin{abstract}
Patricia Janet Padilla-Ornelas*, Ma. del Carmen Martínez-Serna**凶
Padilla-Ornelas, P. J., \& Martínez-Serna, M. C. (2018). Gestión del conocimiento y su influencia sobre el desempeño organizacional en las empresas de Aguascalientes. Investigación y Ciencia de la Universidad Autónoma de Aguascalientes, 26(75), 55-65.
\end{abstract}

\section{RESUMEN}

Este estudio tiene como propósito analizar la influencia de la gestión del conocimiento sobre el desempeño de pequeñas, medianas y grandes organizaciones establecidas en el estado de Aguascalientes. Se realizó una investigación cuantitativa, exploratoria, no experimental a 206 empresas del sector industrial, comercio y servicios. Se aplicaron cuestionarios personales a directivos, gerentes o propietarios de las unidades económicas. El análisis de los datos se realizó mediante la técnica estadística de ecuaciones estructurales (SEM). LOS resultados obtenidos permiten concluir que, si se tiene una cultura que permita políticas y estrategias para gestionar adecuadamente el conocimiento, se entrena a los empleados, mejora en consecuencia el desempeño de las organizaciones. Sin embargo, a los empleados no les gusta compartir sus conocimientos por temor a perder su trabajo.

Palabras clave: gestión del conocimiento; desempeño organizacional; recursos y capacidades.

Keywords: knowledge management; organizational performance; resources and capabilities.

Recibido: 23 de febrero de 2018, aceptado: 6 de agosto de 2018

* Departamento de Comunicación, Centro de Ciencias Sociales y Humanidades, Universidad Autónoma de Aguascalientes. Av. Universidad 940, Ciudad Universitaria, C. P. 20131, Aguascalientes, Aguascalientes, México. Correo electrónico: pjpadill@correo.vaa.mx

** Departamento de Mercadotecnia, Centro de Ciencias Económicas y Administrativas, Universidad Autónoma de Aguascalientes. Av. Universidad 940, Ciudad Universitaria, C. P. 20131, Aguascalientes, Aguascalientes, México. Correo electrónico: mcmartin@correo.vaa.mx

$凶$ Autor para correspondencia
ABSTRACT

The purpose of this study is to analyze the influence of knowledge management on the performance of small, medium and large organizations established in the state of Aguascalientes. Quantitative research was carried out on 206 companies from the industrial, commerce and services sector. The methodology consisted of applying personal questionnaires to chairman, managers or owners of the economic units. The data were analyzed using the statistical technique of modeling structural equations (SEM). The results obtained allow us to conclude that, we have a culture that allows policies and strategies to manage knowledge appropriately, and that if we train employees, therefore the performance of organizations improves. However, employees do not like to share their knowledge because of the fear of losing their jobs.

INTRODUCCIÓN

Una preocupación actual de los empresarios es lograr estrategias efectivas que permitan enfrentar los cambios tecnológicos, económicos y sociales que repercuten en el ámbito competitivo. Buscan desarrollar alternativas que les dejen manejar de forma más efectiva su negocio para obtener mejores resultados; saben que el recurso más valioso del que disponen para lograrlo son sus empleados, por lo que es necesario contar con personas preparadas, competitivas, dispuestas a compartir sus conocimientos en favor de la organización. El Foro Económico Mundial, en inglés World Economic Forum (WEF, 2015), en su reporte de competitividad global, establece que las organizaciones deben hacer frente a los nuevos desafíos recurriendo a 
sus recursos intangibles que se convertirán en los elementos diferenciadores.

El objetivo de esta investigación fue analizar la influencia de la gestión del conocimiento sobre el desempeño de pequeñas, medianas y grandes organizaciones establecidas en el estado de Aguascalientes. La cuestión es cómo mejorar las capacidades organizacionales para hacer que el desempeño de los empleados sea constante y de este modo generar competitividad sostenible en un entorno que cambia constantemente.

Este trabajo está estructurado de la siguiente manera: se realiza un análisis de marco teórico, aborda la teoría de recursos y capacidades para sustentar las variables, el desarrollo conceptual de la GC y el DO, y las dimensiones que conforman cada constructo. Se hace una revisión empírica en empresas del estado de Aguascalientes, se muestran y discuten los resultados. Por último, se presentan las conclusiones y una propuesta para futuras líneas de investigación.

En esta sociedad del conocimiento, como la llama Peter Drucker (1997), pionero en esta teoría, se ha postulado que los medios de producción están cambiando; ya no se concentran en la tierra, trabajo y capital, como se establecía tradicionalmente en la teoría económica. Ese lugar lo ha tomado el conocimiento, la información que posee cada individuo y la forma en que lo utiliza en su organización. Estas están cambiando hacia sistemas basados en el conocimiento, utilizando prácticas de GC para mejorar su competitividad (Guillén, 2000; Mills \& Smith, 2011). De este modo, la gestión del conocimiento (GC) retoma importancia en el quehacer empresarial considerando acciones intangibles, pero con repercusiones importantes en los resultados de la empresa. El término GC se refiere a la creación, evolución y administración del conocimiento desde que se genera hasta que se emplea en una organización. Es un constructo hasta cierto punto poco explorado en la realidad organizacional de México. Sin embargo, a pesar de la importancia de la estrategia de gestión del conocimiento y de las evidencias sobre su contribución al desempeño empresarial, hay poca investigación empírica sobre la elección de las estrategias al respecto (Barroso Tanoira, 2011; Choi \& Lee, 2002; Giampaoli, Ciambotti, \& Bontis, 2017).

En términos conceptuales, de acuerdo con Malhotra, Gosain y Sawy (2005) la GC es un proceso or- ganizacional específico, sistémico, que permite adquirir, organizar y comunicar el conocimiento de un empleado a otro para que este pueda hacer uso de ese conocimiento y lograr mejores resultados; es decir, sean más eficaces y productivos. El conocimiento es un recurso intangible que tiene fundamento en la teoría de los recursos y capacidades al ser una fuente de ventaja competitiva, única y valiosa.

La literatura hace referencia a dos tipos de conocimiento: el tácito (individual) y el explícito (organizacional), cada uno debe favorecer a la creación de la ventaja competitiva; esto es, el conocimiento es un elemento diferenciador que permite a una empresa construir su ventaja competitiva (Navarro de G., 2007). El conocimiento tácito es subjetivo, complejo, relacionado con las percepciones e intuiciones de los empleados, depende de los individuos transmitirlo o compartirlo o no con su organización. Mientras que el conocimiento explícito es fácilmente expresado, capturado y difundido (manuales, libros, etc.), por lo que diversos autores hacen énfasis en la importancia del conocimiento tácito en la creación de la ventaja competitiva (Grant, 1996; Nonaka \& Takeuchi, 1995).

Basados en esta premisa, la GC se ha medido de distintas maneras, considerando diversas fases y actividades; el modelo de Easterby-Smith y Prieto (2008) considera cuatro fases: capturar, elaborar, transmitir, almacenar y compartir el conocimiento. Bozbura (2007) se basa en la propuesta de la Organización para la Cooperación y el Desarrollo Económico, en inglés Organisation for Economic Co-operation and Development (OECD, 2003), que comprende cuatro fases: entrenamiento y dirección de los empleados, políticas y estrategias para gestionar el conocimiento, adquisición y captura del conocimiento externo y la última fase que se refiere a los efectos de la cultura organizacional.

El conocimiento humano cobra importancia en el ámbito de los negocios y del desarrollo social (Nonaka \& Takeuchi, 1995) y lo hace con una finalidad: mejorar los resultados de la organización. Las empresas sin importar su sector de actividad o tamaño buscan obtener el máximo beneficio, así, el desempeño organizacional (DO) es un factor clave, se considera prioritario en términos de rendimiento y logro de resultados, a partir de esta premisa se implementan distintas estrategias. Si bien no existe un consenso sobre la definición de DO, es un tema importante para investigadores y empresarios. Ca- 
misón Zornoza y Cruz Ros (2008) hacen referencia a que estas diferencias sobre el contenido, las dimensiones para medirlo no han permitido su consolidación teórica y empírica. A pesar de estas diferencias, la literatura señala que se aborda desde dos perspectivas: una general, partiendo de la teoría de la administración, o bien, particular, considerando la teoría de la estrategia.

Esta investigación retoma la propuesta de Wu y Chen (2014), al considerar acciones integrales de la organización como el nivel de satisfacción de sus clientes, el desempeño productivo de la organización, la calidad de sus productos y las utilidades percibidas.

Existe evidencia de que el desempeño mejora si se gestiona y aplica el conocimiento que se genera en la organización (Barroso Tanoira \& Córdova Buenfil, 2013; Darroch, 2005; Estrada \& Dutrénit, 2007, Giampaoli et al., 2017). El conocimiento puede generarse al interior de la empresa o se adquiere del exterior; no importa cómo se construya, si se gestiona adecuadamente facilita la alineación de los procesos, el logro de objetivos y la consecución de los resultados. La evidencia empírica muestra que los recursos intangibles favorecen el desempeño competitivo (Estrada \& Dutrénit, 2007). Investigaciones previas señalan que para obtener buenos resultados no solo dependen de la correcta utilización de sus activos físicos, sino también de la adecuada gestión de los recursos y capacidades (Lee \& Sukoco, 2007; Mills \& Smith, 2011).

Wu y Chen (2014) hacen una propuesta para medir el desempeño organizacional como consecuencia de la GC. Los estudios empíricos han encontrado poca o ninguna mejora a pesar de grandes inversiones en sistemas de $\mathrm{Tl}$, rediseños de procesos internos y externos (Bogner \& Bansal, 2007; Kulkarni, Ravindran, \& Freeze, 2006). El desempeño organizacional también se define como una construcción formativa con los cuatro indicadores de desempeño: excelencia operacional, liderazgo de producto, satisfacción del cliente y logro financiero (Wu \& Chen, 2014). Tanto la GC como el DO se han posicionado como conceptos importantes en los últimos 15 años; por tanto, se basa en la teoría de los recursos y capacidades (RBV) al considerar elementos en su mayoría intangibles. No podemos hablar de RBV sin considerar el trabajo de Barney (1991), donde señala que cada organización posee recursos y capacidades de conocimiento, raros, valiosos y no sustituibles, que son los que utiliza para lograr una ventaja competitiva sostenible.

Si se toman como referencia los trabajos de Penrose (1959), así como de Nelson y Winter (1982), sus postulados permiten argumentar que el conocimiento es un recurso en sí mismo, que si se gestiona de manera adecuada permitirá hacer mejor uso de los recursos disponibles de la compañía y, en consecuencia, convertir esos recursos en capacidades. La importancia de cada uno de estos elementos intangibles ha favorecido el logro de importantes cambios en las empresas actuales que han permitido mejorar la oferta de bienes y servicios capaces de crear ventajas competitivas. Cuantos más recursos intangibles tenga una empresa mayor será su posibilidad de crear valor y, con ello, lograr estas ventajas competitivas (Lev, 2004; Seeley \& Dietrich, 2000).

En la literatura se aborda el vínculo de gestión del conocimiento con el desempeño organizacional que mostró resultados empíricos positivos, como en Darroch (2005), en México Estrada y Dutrénit (2007), Giampaoli et al. (2017) y Migdadi, Zaid, Yousif y Almestarihi (2018). Los resultados señalan que las prácticas de GC favorecen la calidad de conocimiento que se genera, así como la integración de empleados y socios de la cadena de suministro, lo que conduce a un mejor desempeño organizacional. Diversos estudios a nivel internacional han dado muestra de la importancia de la GC, cuyos hallazgos han permitido corroborar que las empresas que implementan dichas prácticas obtienen mejores resultados en comparación con sus competidores (Palacios Marqués \& Garrigós Simón, 2006; Wu \& Hu, 2012). Basados en estas investigaciones se plantea la hipótesis a evaluar:

$\mathrm{H}_{1}$ : La gestión del conocimiento influye de manera positiva y significativa en el desempeño organizacional en las empresas del estado de Aguascalientes. 


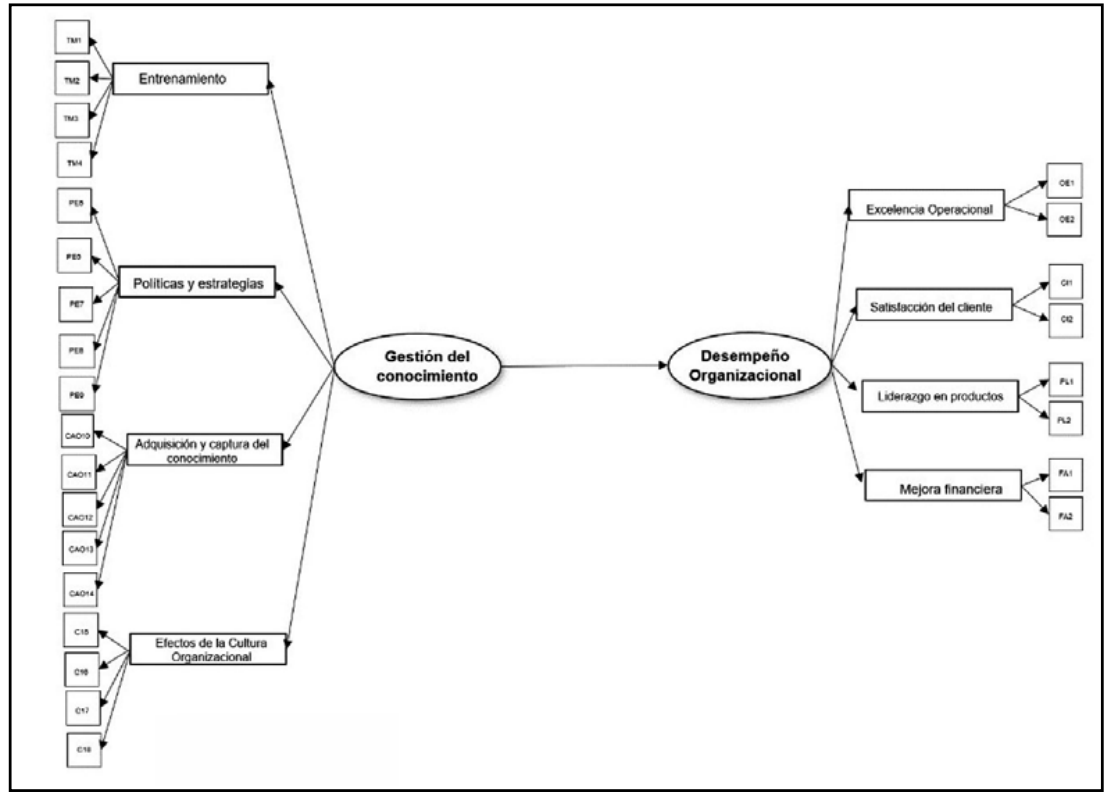

Figura 1. Modelo de investigación. Elaboración propia.

En la figura 1 se puede observar el modelo de investigación.

El modelo está conformado por dos constructos de investigación; el primero, GC que se mide a partir de cuatro dimensiones y se relaciona con el DO, que se mide con cuatro dimensiones, mismas que se explicarán más adelante.

\section{MATERIALES Y MÉTODOS}

La presente investigación es cuantitativa, utiliza la encuesta para obtener información y medir ambos constructos. El estudio es ubicado en un nivel organizacional; es decir, la información que se obtiene es a partir de la percepción de los gerentes o directores de las empresas sujeto de estudio. Se desarrolló un muestreo por conveniencia dado que este método de acuerdo con Rodríguez, Pierdant y Rodríguez (2014) se aplica cuando la opinión sobre un tema es de suma importancia, por lo que los sujetos de investigación deben cumplir ciertos criterios, donde los participantes tuvieran conocimiento del tema y tomaran decisiones en sus empresas. Sin embargo, Rodríguez et al. (2014) advierten que bajo este método las muestras no son representativas, por lo que no es correcto hacer inferencias de la población total.

El trabajo de campo se llevó a cabo durante 7 meses, en el periodo comprendido desde enero hasta julio de 2017. Los cuestionarios se entregaron personalmente a 300 directores o propietarios de las empresas. Con cada cuestionario se incluyó una carta de presentación y confidencialidad sobre el tratamiento y difusión de los datos (resultados). Se dio seguimiento telefónico y se recogieron de manera personal. El margen de respuesta fue de $68 \%$, esto equivale a 206 empresas, 72 pertenecen al sector Industrial. Las características que debían reunir para ser consideradas en el estudio fueron tener más de 10 empleados, estar en funcionamiento y ubicadas en el estado de Aguascalientes, México.

Los programas informáticos de elección fueron el SPSS 23 y el SPSS AMOS (Analysis of Moment Structures). El instrumento está conformado por dos escalas que han sido probadas y validadas en otros contextos. Para medir gestión del conocimiento se utilizó la escala de Bozbura (2007) y para medir desempeño organizacional la propuesta por Wu y Chen (2014), que fue diseñada específicamente para medir el impacto de la GC. Se utilizó una escala tipo Likert de siete posiciones que van desde (1) total desacuerdo hasta (7) totalmente de acuerdo.

En cuanto a la gestión del conocimiento se evaluó la forma en que se genera, adquiere y transmite el conocimiento en la empresa a partir de 18 indicadores, divididos en cuatro dimensiones: entrenamiento y dirección de los empleados (cuatro ítems), políticas y estrategias para gestionar 
el conocimiento (cinco ítems), adquisición y captura del conocimiento externo (cinco ítems) y la última dimensión se refiere a los efectos de la cultura organizacional (cuatro ítems). Asimismo, se midió el desempeño organizacional desde el punto de vista subjetivo a partir de cuatro dimensiones y ocho ítems. Los encuestados contestaron a partir de su experiencia cómo ha sido su desempeño productivo (dos ítems), el nivel de satisfacción de sus clientes (dos ítems), la calidad de sus productos (dos ítems), así como las utilidades o ingresos percibidos (dos ítems).

Para analizar los datos se recurrió a distintas pruebas para determinar validez y fiabilidad del instrumento. De acuerdo con Vila López, Küster Boluda y Aldás Manzano (2000), una escala debe contener dos propiedades psicométricas: fiabilidad y validez. La primera es necesaria para el uso de un instrumento. Haertel (2006) refiere que la fiabilidad se determina por los valores observados en una variable aleatoria consistente en todas las posibles puntuaciones que se consiguen de forma repetida en la recolección de la información. Prieto y Delgado (2010) refieren la importancia de tener en cuenta que la fiabilidad de un instrumento se puede ver afectada por factores como la población o las condiciones en las que se realice la investigación.

Uno de los índices más recurrentes para determinar la fiabilidad es el valor del $\alpha$ de Cronbach, que de acuerdo con Hair, Black, Babin y Anderson (2010) debe ser mayor que 0.50. Si bien esta es la prueba más usual, no es contundente, puesto que no considera la influencia del resto de variables. Con la finalidad de corroborar y asegurar la consistencia interna de los datos se realizaron otras pruebas de fiabilidad para cada una de las escalas. Fornell y Larcker (1981) proponen un cálculo adicional cuando se tienen dos o más variables en el modelo, proponen el Índice de Fiabilidad Compuesta (IFC) con valores por encima del 0.70 para cada variable, así como el Índice de la Varianza Extraída (IVE), cuyo valor debe ser igual o mayor que 0.50. Para esta investigación se utilizan los tres índices para medir la fiabilidad de escalas.

Se verificó la validez del instrumento de la siguiente forma: la validez convergente mediante el análisis de cargas factoriales, que deben ser significativas y estar por arriba de 0.70 (Anderson
\& Gerbing, 1988). Posteriormente, se comprobó la discriminante mediante el test de la Varianza Extraída del instrumento de recolección de datos, tomando como criterio que el IVE de los dos factores debe ser mayor que el cuadrado de la correlación entre dichos factores. En este estudio se utilizó el modelado de ecuaciones estructurales (SEM) para analizar la relación de GC-DO. El método SEM permite un análisis de factores, de covarianzas y el análisis factorial confirmatorio. Además, este método combina la información de causa-efecto con datos estadísticos, de los que si son significativos se puede precisar que la construcción teórica es válida; es decir, proporciona guías para la aplicación del modelo a la realidad.

Se aplicó el modelado de ecuaciones estructurales (SEM) para poder determinar la influencia que ejerce la gestión del conocimiento en el desempeño de las empresas en el estado de Aguascalientes $y$, de esta manera, establecer de acuerdo con el ajuste del modelo qué tanto la teoría investigada está representada en la realidad de la muestra.

Los resultados de las pruebas de confiabilidad y validez convergente de este estudio se encuentran en la tabla 1. Para determinar la fiabilidad, los valores de alfa de Cronbach $(\alpha)$ para cada una de las escalas gestión del conocimiento y desempeño organizacional son superiores a .591, por lo que las mismas cumplen con el requisito establecido por Bernstein y Nunnally (1994), lo que demuestra consistencia interna. Se desarrolló una segunda prueba para verificar la fiabilidad de las escalas a utilizar, los resultados de la prueba IFC (ver tabla 1), los cuales fueron superiores a 0.7, lo que cumple con los rangos establecidos por la teoría de Fornell y Larcker (1981).

El test de la validez convergente se llevó a cabo mediante la comprobación de cargas factoriales con valores iguales o mayores a 0.7 (Anderson \& Gerbing, 1988). El análisis de cargas factoriales en cada dimensión permite corroborar cargas factoriales superiores a .70, por lo que los valores cumplen con el requisito, de igual manera, así se determina que cada ítem mide lo que tiene que medir.

La validez discriminante se determinó mediante el test de la varianza extraída, cuyos resultados se pueden observar en la tabla 2. 


\section{IIVESTIGACIÓn Y CIECGIA DE \\ LA UNIVERSIDAD AUTÓNOMA \\ DE AGUASCALIERTES

Tabla 1

Prueba de fiabilidad y validez convergente para el modelo de investigación

\begin{tabular}{|c|c|c|c|c|c|c|c|}
\hline Constructo & Dimensión & Ítems & $\begin{array}{c}\text { Alfa de } \\
\text { Cronbach } \\
\text { (Dimensión) }\end{array}$ & $\begin{array}{l}\text { Carga } \\
\text { Factorial }\end{array}$ & $\begin{array}{l}\text { Valor } t \\
\text { robusto }\end{array}$ & $\begin{array}{l}\text { índice de } \\
\text { Fiabilidad } \\
\text { Compuesta } \\
\text { (IFC) }\end{array}$ & $\begin{array}{l}\text { Índice de } \\
\text { la Varianza } \\
\text { Extraída } \\
\text { (IVE) }\end{array}$ \\
\hline \multirow{18}{*}{ 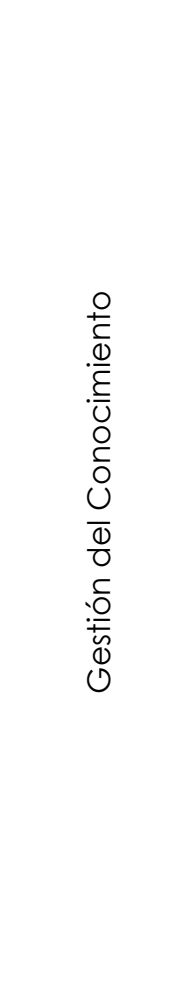 } & \multirow{4}{*}{ Entrenamiento } & TM1 & \multirow{18}{*}{0.891} & $0.898^{* * *}$ & $1.000^{*}$ & \multirow{4}{*}{0.906} & \multirow{4}{*}{0.762} \\
\hline & & TM2 & & $0.864^{* * *}$ & 19.311 & & \\
\hline & & TM3 & & $0.817^{* * *}$ & 20.735 & & \\
\hline & & TM4 & & $0.723^{* * *}$ & 24.671 & & \\
\hline & \multirow{5}{*}{ Políticas y Estrategias } & PE5 & & $0.819^{* * *}$ & $1.000 *$ & \multirow{5}{*}{0.925} & \multirow{5}{*}{0.861} \\
\hline & & PE6 & & $0.882^{* * *}$ & 12.861 & & \\
\hline & & PE7 & & $0.713^{* * *}$ & 10.454 & & \\
\hline & & PE8 & & $0.878^{* * *}$ & 22.895 & & \\
\hline & & PE9 & & $0.796^{* * *}$ & 17.826 & & \\
\hline & \multirow{5}{*}{$\begin{array}{l}\text { Adquisición y Captura } \\
\text { del Conocimiento }\end{array}$} & CAO10 & & $0.814^{* * *}$ & $1.000 *$ & \multirow[b]{5}{*}{0.911} & \multirow{5}{*}{0.773} \\
\hline & & CAO11 & & $0.821^{* * *}$ & 8.567 & & \\
\hline & & $\mathrm{CAO} 12$ & & $0.852^{* * *}$ & 4.561 & & \\
\hline & & $\mathrm{CAO} 13$ & & $0.798^{* * *}$ & 15.972 & & \\
\hline & & $\mathrm{CAO} 14$ & & $0.717^{* * *}$ & 11.087 & & \\
\hline & \multirow{4}{*}{$\begin{array}{l}\text { Efectos de la cultura } \\
\text { organizacional }\end{array}$} & C15 & & $0.731^{* * *}$ & $1.000^{*}$ & \multirow{4}{*}{0.815} & \multirow{4}{*}{0.596} \\
\hline & & $\mathrm{C} 16$ & & $0.816^{* * *}$ & 6.875 & & \\
\hline & & $\mathrm{C} 17$ & & $0.754^{* * *}$ & 7.342 & & \\
\hline & & C18 & & $0.823^{* * *}$ & 13.987 & & \\
\hline \multirow{8}{*}{ 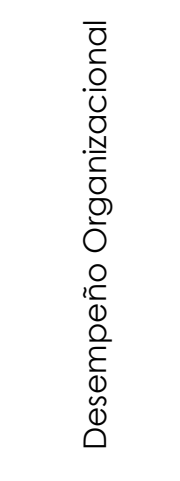 } & \multirow{2}{*}{ Excelencia Operacional } & EO1 & & $0.836^{* * *}$ & $1.000 *$ & \multirow[b]{2}{*}{0.882} & \multirow{2}{*}{0.789} \\
\hline & & EO2 & & $0.854^{* * *}$ & 25.733 & & \\
\hline & \multirow{2}{*}{ Satisfacción del Cliente } & $\mathrm{Cll}$ & & $0.817^{* * *}$ & $1.000 *$ & \multirow{2}{*}{0.873} & \multirow{2}{*}{0.707} \\
\hline & & $\mathrm{Cl} 2$ & P 0 & $0.931^{* * *}$ & 24.547 & & \\
\hline & \multirow{2}{*}{ Liderazgo en Productos } & PL1 & & $0.895^{* * *}$ & $1.000 *$ & \multirow{2}{*}{0.905} & \multirow{2}{*}{0.623} \\
\hline & & PL2 & & $0.912^{* * *}$ & 12.388 & & \\
\hline & \multirow{2}{*}{ Mejora Financiera } & FAl & & $0.896^{* * *}$ & $1.000 *$ & \multirow{2}{*}{0.887} & \multirow{2}{*}{0.702} \\
\hline & & FA2 & & $0.839^{* * *}$ & 24.368 & & \\
\hline
\end{tabular}

Nota: Medidas de bondad de ajuste: Chi $(g l)=290.582$ (136); RMSEA =.0.39; NFI= .907; $C F I=.904 ; R M R=.030 ;$ SRMR= .052. Valores significativos: ${ }^{*}=p$ $<0.05 ; * *=0.01 ;{ }^{* * *}=p<0.001$. 
Tabla 2

Prueba de validez discriminante de las escalas utilizadas en el modelo

\begin{tabular}{|c|c|c|c|c|c|c|c|c|}
\hline & $\begin{array}{c}\text { Entrena- } \\
\text { miento }\end{array}$ & $\begin{array}{l}\text { Políticas y } \\
\text { Estrategias }\end{array}$ & $\begin{array}{l}\text { Adquisición y } \\
\text { Captura del } \\
\text { conocimiento }\end{array}$ & $\begin{array}{l}\text { Efectos de } \\
\text { la cultura } \\
\text { organiza- } \\
\text { cional }\end{array}$ & $\begin{array}{l}\text { Excelencia } \\
\text { operacional }\end{array}$ & $\begin{array}{c}\text { Satisfacción } \\
\text { del } \\
\text { cliente }\end{array}$ & $\begin{array}{l}\text { Liderazgo } \\
\text { en } \\
\text { productos }\end{array}$ & $\begin{array}{c}\text { Mejora } \\
\text { financiera }\end{array}$ \\
\hline 1. Entrenamiento & 0.762 & & & & & & & \\
\hline $\begin{array}{l}\text { 2. Políticas y } \\
\text { Estrategias }\end{array}$ & 0.380 & 0.861 & & & & & & \\
\hline $\begin{array}{l}\text { 3. Adquisición } \\
\text { y Captura del } \\
\text { conocimiento }\end{array}$ & 0.296 & 0.349 & 0.773 & & & & & \\
\hline $\begin{array}{l}\text { 4. Efectos de } \\
\text { la Cultura } \\
\text { Organizacional }\end{array}$ & 0.311 & 0.372 & 0.361 & 0.596 & & & & \\
\hline $\begin{array}{l}\text { 5. Excelencia } \\
\text { operacional }\end{array}$ & 0.357 & 0.301 & 0.396 & 0.466 & 0.789 & & & \\
\hline $\begin{array}{l}\text { 6. Satisfacción } \\
\text { del Cliente }\end{array}$ & 0.305 & 0.298 & 0.246 & 0.383 & 0.301 & 0.707 & & \\
\hline $\begin{array}{l}\text { 7. Liderazgo en } \\
\text { productos }\end{array}$ & 0.282 & 0.195 & 0.139 & 0.189 & 0.103 & 0.096 & 0.623 & \\
\hline $\begin{array}{l}\text { 8. Mejora } \\
\text { financiera }\end{array}$ & 0.196 & 0.127 & 0.103 & 0.111 & 0.166 & 0.101 & 0.203 & 0.702 \\
\hline
\end{tabular}

Nota: Elaboración por parte de los autores a partir de los datos de la muestra.

En la diagonal se encuentran los valores obtenidos para el IVE de cada dimensión, en la parte inferior se muestran valores superiores de la covarianza entre las dimensiones donde no debe existir la unidad; es decir, valor igual a 1 (Anderson \& Gerbing, 1988). Los resultados permiten comprobar que cada uno es menor al IVE, así se establece que las dimensiones no ejercen influencia entre sí, miden lo que les corresponde.

Después de comprobar la fiabilidad y validez de las escalas se procedió a verificar el ajuste del modelo, los índices aplicados a esta investigación para medir la bondad de ajuste son, en cuanto a Índices absolutos: RMR, SRMR, RMSEA y la razón $\mathrm{Chi}^{2}$ entre los grados de libertad (Chi $\left./ \mathrm{gl}\right)$, así como los índices relativos o de ajuste incremental, que constituyen una comparación entre la $\mathrm{Chi}^{2}$ del modelo propuesto y la de un modelo nulo en el que las variables no tienen relación entre sí, estos índices son: NFI, NNFI y CFI. Para determinar la bondad de ajuste del modelo, el RMR, cuyo valor debe ser cercano a 0 para indicar un buen ajuste y el SRMR debe estar entre 0 y 1 (Hu \& Bentler, 1995).
El valor obtenido con los datos de la muestra para el modelo estructural es un $\mathrm{Chi}^{2} / \mathrm{gl}$ igual a 2.13, lo que de acuerdo con Marsh y Hocevar (1985) indica que el modelo tiene un ajuste razonable. Para el índice RMR se consiguió un valor de .030 y de .052 para el SRMR; ambos índices están dentro del rango establecido como bueno, por lo que hay un buen ajuste que explica las correlaciones en un rango de error promedio de .052.

Uno de los índices más contundentes para explicar el ajuste del modelo es RMSEA (Root Mean Square Error of Aproximation), ya que conduce a conclusiones acertadas en relación con la calidad del modelo, para esta investigación el RMSEA tiene un valor de .039.

Los valores para el modelo teórico de investigación están en .900 para el NFI, .907 para el índice NNFI (TLI) y .904 el valor del CFI, lo cual indica que todos los índices están dentro del rango establecido de más de 9 (Bentler, 1990; Byrne, 2013), por tanto, el modelo de investigación tiene un buen ajuste. 
Tabla 3

Resultados del modelo global ajustado

\begin{tabular}{|c|c|c|c|c|}
\hline Hipótesis & $\begin{array}{l}\text { Relación } \\
\text { estructural }\end{array}$ & $\begin{array}{c}\text { Coeficiente } \\
\text { estandarizado }\end{array}$ & $\begin{array}{l}\text { Valor } t \\
\text { robusto }\end{array}$ & $R^{2}$ \\
\hline $\begin{array}{l}\mathrm{H}_{1} \text { : Existe evidencia suficiente para afirmar } \\
\text { que la gestión del conocimiento influye } \\
\text { en el desempeño organizacional de las } \\
\text { empresas del estado de Aguascalientes. }\end{array}$ & $\mathrm{GC} \rightarrow \mathrm{DO}$ & .796 *** & 6.79 & .323 \\
\hline
\end{tabular}

RESULTADOS

Una vez que se verificó la bondad de ajuste del modelo de ecuaciones estructurales se analizó la relación estructural de GC-DO y los resultados obtenidos se presentan en la tabla 3.

De acuerdo con los datos obtenidos y presentados en la tabla de resultados (tabla 3), el valor de la carga factorial estandarizada que relaciona la variable de GC con la variable de DO es de .796 y existe un valor $t$ robusto de 6.79, el cual es significativo al 0.001 . Se obtuvo un coeficiente de determinación $\left(R^{2}\right)$ de .323 , el cual demuestra una relación causal; es decir, que la GC tiene efectos positivos y significativos sobre el DO en las empresas de Aguascalientes. Por ello se acepta la hipótesis planteada $H_{1}$ : La gestión del conocimiento influye de manera positiva y significativa en el desempeño organizacional en las empresas del estado de Aguascalientes.

Las medidas de bondad de ajuste están en el rango de los parámetros aceptados como buenos; es decir, que la teoría está bien representada en la realidad de la muestra. Los hallazgos de esta investigación permiten establecer que la GC contribuye de manera positiva y significativa con el desempeño general de las empresas.

\section{DISCUSIÓN}

Una de las aportaciones más significativas del trabajo de Penrose (1959) está basada en considerar lo que sucede dentro de la empresa, precisamente la perspectiva de este trabajo. La gestión del conocimiento se lleva a cabo mediante el aprendizaje individual, es un proceso organizacional que permite adquirir, organizar y comunicar el conocimiento de un empleado a otro para que estos puedan hacer uso de ese conocimiento y obtener mejores resultados. Se corrobora lo estipulado en otras investigaciones que establecen una relación positiva entre GC y DO (Darroch, 2005; Estrada \& Dutrénit, 2007; Giampaoli et al., 2017; Migdadi et al., 2018).

De acuerdo con los resultados de esta investigación cada empresa posee una serie de recursos y capacidades de conocimiento; sin embargo, no los tiene plenamente identificados por lo que no desarrolla al máximo sus potencialidades, así que debe dedicar esfuerzos a identificar e implementar más prácticas de GC, también a mejorar las capacidades del proceso empresarial y el aprendizaje organizacional para lograr un mejor desempeño (figura 2).

En la presente investigación se ha reflexionado sobre la importancia de los factores intangibles, específicamente del conocimiento y se ha probado estadísticamente que favorece el desempeño organizacional, existe evidencia de que no ha sido así en todos los contextos. Bogner \& Bansal (2007) encontraron poca mejoría en esta relación GC-DO.

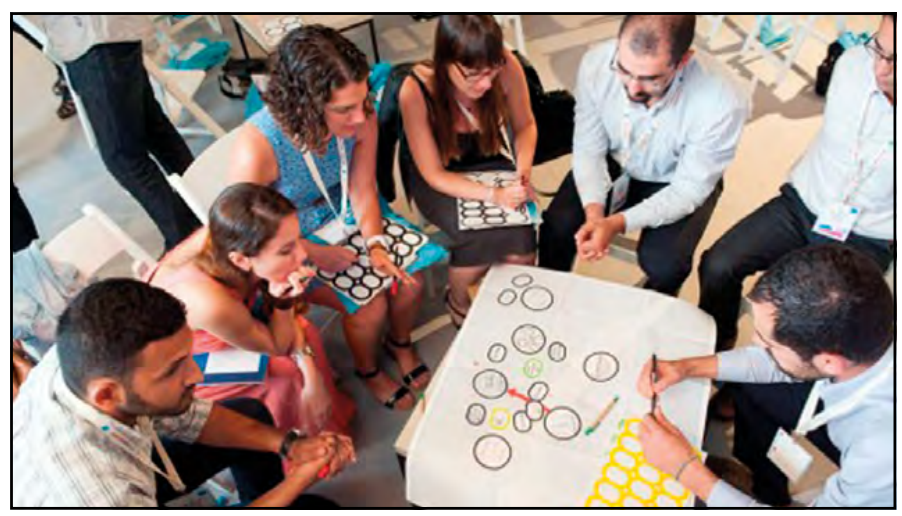

Figura 2. Grupo de trabajo en el Smart Aquae Campus, de la Fundación Aquae.

Imagen tomada de El País (21 de mayo de 2016). 
En otra investigación realizada por Kulkarni et al. (2006) se establece que no hay evidencia de que la GC mejore el desempeño a pesar de grandes inversiones en sistemas de $\mathrm{Tl}$, rediseños de procesos internos y externos. Sin embargo, el número de investigaciones empíricas con evidencia de que la GC mejora el DO es mayor (Palacios Marqués \& Garrigós Simón, 2006; Wu \& Hu, 2012), por lo que es importante aprender a gestionar adecuadamente el conocimiento en las organizaciones.

\section{CONCLUSIONES}

Una vez revisada la teoría y los datos obtenidos mediante las encuestas, fue posible realizar las pruebas de fiabilidad y validez de escalas para determinar la relación directa entre gestión del conocimiento y desempeño organizacional, con escalas propuestas por Bozbura (2007) y Wu y Chen (2014), respectivamente. Es posible concluir que tanto el desempeño organizacional como la gestión del conocimiento se derivan de las prácticas diarias, de acciones, políticas y condiciones en las que se desarrolla la empresa; si la misma está enfocada en sus empleados, su entrenamiento, estrategias y una cultura que favorezca el conocimiento, tendrá un impacto favorable sobre el desempeño financiero, la satisfacción del cliente y la función general de la organización.

La gestión del conocimiento y sus dimensiones (entrenamiento y dirección de los empleados, políticas y estrategias para gestionar el conocimiento, adquisición y captura del conocimiento externo, los efectos de la cultura organizacional) mostraron significancia con el desempeño organizacional de las PyME; esto es, si la empresa gestiona el conocimiento, si entrena, dirige a sus empleados y genera una cultura en la que se generen y compartan los conocimientos, tiene un impacto positivo en el desempeño operacional, la satisfacción del cliente, el liderazgo en productos y, en consecuencia, en el desempeño financiero (Bozbura, 2007; Wu \& Chen, 2014).

Los resultados han respaldado la propuesta de Bozbura (2007), la GC parte del entrenamiento y di- rección del personal; para poder crear nuevo conocimiento, compartir y capitalizar es importante la cultura de cada organización. Es decir, debe favorecer la flexibilidad y capacidad de adaptación a los cambios constantes, no centrados únicamente en las personas, sino también en los procesos y tecnología (Bhatt, 2001). Esta visión contribuye a que el conocimiento se gestione de forma más efectiva.

La investigación empírica muestra que los estudios sobre gestión del conocimiento pueden ser de mucha utilidad en la comprensión del funcionamiento de las organizaciones. Sin embargo, este estudio demuestra que existe una gran necesidad de realizar más estudios que permitan comprender mejor la manera en que el conocimiento se origina, transforma, comparte y aplica en las PyME, así como las prácticas de gestión del conocimiento que se realizan en cada empresa. Por ello se sugiere que las organizaciones deben tener mayor conciencia de la importancia de las cuestiones de GC, su manejo y el impacto que pueden generar en los resultados del negocio. Asimismo, es importante considerar que existe poca literatura con referencia a lo que es o significa gestión efectiva del conocimiento y sobre resultados cuantificables de la gestión del conocimiento sobre el desempeño de las compañías.

Este trabajo contribuye a la escasa investigación empírica sobre la gestión del conocimiento dentro de las empresas en el contexto nacional, además de la repercusión que tiene sobre el desempeño de las organizaciones cuando existe un programa coordinado y bien implementado sin importar el tamaño o tipo de organización. Esta investigación considera una muestra de un estado en particular, por lo que es importante replicar el estudio en otros contextos, tomar en cuenta los factores externos que repercuten en la forma en que las empresas gestionan el conocimiento. En el contexto que vivimos la inestabilidad laboral dificulta la transmisión del conocimiento, hay rotación constante y algunas políticas que castigan o desprecian la experiencia, por lo que es necesario analizar la repercusión de la GC en los sistemas de contratación. 
- Anderson, J. C., \& Gerbing, D. W. (1988). Structural equation modeling in practice: A review and recommended twostep approach. Psychological Bulletin, 103(3), 411-423. doi: 10.1037/0033-2909.103.3.411

- Barney, J. (1991). Firm resources and sustained competitive advantage. Journal of Management, 17(1), 99-120. doi: $10.1177 / 014920639101700108$

- Barroso Tanoira, F. G. (2011). Gestión del conocimiento en empresas y organizaciones sociales productivas exitosas en el estado de Yucatán. XVI Congreso Internacional de Contaduría, Administración e Informática en la UNAM. Recuperado de http://congreso.investiga.fca.unam.mx/ docs/xvi/docs/1M.pdf

conocimiento y desempeño organizacional en micro y pequeñas empresas de poblaciones del interior del estado de Yucatán. Avance de investigación. XVIII Congreso Internacional de Contaduría Administración e Informática. Recuperado de http://congreso.investiga.fca.unam.mx/ docs/xviii/docs/1.13.pdf

- Bentler, P. M. (1990). Comparative fix indexes in structural models. Psychological Bulletin, 107(2), 238-246. doi:10.1037/0033-2909.107.2.238

- Bernstein, I. H., \& Nunnally, J. C. (1994). Psychometric Theory (3. ed.). New York, NY, US: McGraw-Hill.

- Bhatt, G. D. (2001). Knowledge management in organizations: Examining the interaction between technologies, techniques, and people. Journal of Knowledge Management, 5(1), 68-75. doi: 10.1108/13673270110384419

- Bogner, W. C., \& Bansal, P. (2007). Knowledge management as the basis of sustained high performance. Journal of Management Studies, 44(1), 165-188.

- Bozbura, F. T. (2007). Knowledge management practices in Turkish SMEs. Journal Enterprise information Management, 20(2), 209-221.

- Byrne, B. M. (2013). Structural equation modeling with AMOS: Basic concepts, applications, and programming (2nd. ed.). NY, US: Routledge.

- Camisón Zornoza, C., \& Cruz Ros, S. (2008). La medición del desempeño organizativo desde una perspectiva estratégica: Creación de un instrumento de medida. Revista Europea de Dirección y Economía de la Empresa, 17(1), 79-102.

- Choi, B., \& Lee, H. (2002). Knowledge management strategy and its link to knowledge creation process. Expert Systems with Applications, 23(3), 173-187.
- Darroch, J. (2005) Knowledge management, innovation and firm performance. Journal of Knowledge Management, 9(3), 101-115. doi: 10.1108/13673270510602809

- Drucker, P. (1997). The future that has already happened. Harvard Business Review, September-October.

- Easterby-Smith, M., \& Prieto, I. M. (2008). Dynamic capabilities and knowledge management: An integrative role for learning? British Journal of Management, 19(3), 235-249.

- El País. (21 de mayo de 2016). Grupo de trabajo en el Smart Aquae Campus, de la Fundación Aquae [Fotografía]. Recuperada de https://elpais.com/economia/2016/05/19/ actualidad/1463645912_860639.html

- Estrada, S., \& Dutrénit, G. (2007). Gestión del conocimiento en pymes y desempeño competitivo. Engevista, 9(2), 129-148.

- Fornell, C., \& Larcker, D. F. (1981). Evaluating structural equation models with unobservable variables and measurement error. Journal of Marketing Research, 18(1), 39-50. doi: $10.2307 / 3151312$

- Giampaoli, D., Ciambotti, M., \& Bontis, N. (2017). Knowledge management, problem solving and performance in top Italian firms. Journal of Knowledge Management, 21 (2), 355-375. doi: 10.1108/JKM-03-2016-0113

- Grant, R. M. (1996). Toward a knowledge-based theory of the firm. Strategic Management Journal, 17(S2), 109-122.

- Guillén, M. F. (2000). Business groups in emerging economies: A resource-based view. The Academy of Management Journal, $43(3), 362-380$.

- Haertel, E. H. (2006). Reliability. En R. L. Brennan (Ed.) Educational Measurement (pp. 65-110). Westport, CT: American Council on Education-Praeger Publishers.

- Hair, J. F., Black, W. C., Babin, B. J., \& Anderson, R. E. (2010). Multivariate Data Analysis. England: Pearson.

- Hu, L., \& Bentler, P. M. (1995). Evaluating model fit. En R. H. Hoyle (Ed.), Structural equation modeling. Concepts, issues, and applications (pp. 76-99). CA, US: SAGE Publications.

- Kulkarni, U. R., Ravindran, S., \& Freeze, R. (2006). A knowledge management success model: Theoretical development and empirical validation. Journal of Management Information Systems, 23(3), 309-347.

- Lee, L. T. S., \& Sukoco, B. M. (2007). The effects of entrepreneurial orientation and knowledge management capability on organizational effectiveness in Taiwan: The moderating role of social capital. International Journal of Management, 24 (3), 549-573. 


\section{IIVESTIGAGIÓn Y CUERCIA DE LA UחIVERSIDAD AUTÓNOMA DE AGUASCALIERTES}

- Lev, B. (2004). Sharpening the intangibles edge. Harvard Business Review, June, 109-116.

- Malhotra, A., Gosain, S., \& Sawy, O. A. (2005). Absorptive capacity configurations in supply chains: Gearing for partnerenabled market knowledge creation. MIS Quarterly, 29(1), 145-187.

- Marsh, H. W., \& Hocevar, D. (1985). Application of confirmatory factor analysis to the study of self-concept: First-and higher order factor models and their invariance across groups. Psychological Bulletin, 97(3), 562-582. doi: 10.1037/00332909.97.3.562

- Migdadi, M. M., Zaid, M. K. S. A., Yousif, M., \& Almestarihi, R. D. (2018). An empirical examination of collaborative knowledge management practices and organisational performance: The mediating roles of supply chain integration and knowledge quality. International Journal of Business Excellence, 14(2), 180211.

- Mills, A. M., \& Smith, T. A. (2011). Knowledge management and organizational performance: A decomposed view. Journal of Knowledge Management, 15(1), 156-171. doi: $10.1108 / 13673271111108756$

- Navarro de G., K. (2007). Aproximación estratégica a la gestión del conocimiento de las empresas familiares (Anteproyecto de tesis doctoral). Universidad de Castilla-La Mancha, España.

- Nelson, R. R., \& Winter, S. G. (1982). An evolutionary theory of economic change. Cambridge, MA, US: Harvard University Press.

- Nonaka, I., \& Takeuchi, H. (1995). The knowledge-creating company. How japanese companies create the dynamics of innovation. New York, NY, US: Oxford University Press.

- Organisation for Economic Co-operation and Development. (2003). Measuring knowledge management in the business sector: First steps. Ottawa, CA: OECD.
- Palacios Marqués, D., \& Garrigós Simón, F. J. (2006). The effect of knowledge management practices on firm performance. Journal of Knowledge Management, 10(3), 143-156.

- Penrose, E. (1959). The theory of the growth of the firm. Oxford, UK: Oxford University Press.

- Prieto, G., \& Delgado, A. R. (2010). Fiabilidad y validez. Papeles del psicólogo, 31 (1), 67-74.

- Rodríguez, J., Pierdant, A., \& Rodríguez, C. (2014). Estadística para administración. México: Grupo Editorial Patria. Recuperado de https://ebookcentral.proquest.com

- Seeley, C., \& Dietrich, B. (2000). Crafting a knowledge management strategy. Knowledge Management Review, 3 , $18-21$.

- Vila López, N., Küster Boluda, I., \& Aldás Manzano, J. (2000). Desarrollo y validación de escalas de medida en marketing (73 pp.) [Reporte]. España: Universitat de Valencia. Recuperado de https://www.researchgate.net/publication/267725602_ Desarrollo_y_validacion_de_escalas_de_medida_en_ Marketing

- World Economic Forum. (2015). The global competitiveness report 2015-2016. Geneva, Switzerland: World Economic Forum.

- Wu, I. L., \& Chen, J. L. (2014). Knowledge Management driven firm performance: The roles of business process capabilities and organizational learning. Journal of Knowledge Management, 18(6), 1141-1164.

- Wu, I.L., \&HU, Y.P. (2012). Examining knowledge management enabled performance for hospital professionals: A dynamic capability view and the mediating role of process capability. Journal of the Association for Information Systems, 13(12), 976-999. 